\title{
Digalactosyl-diacylglycerol-deficiency lowers the thermal stability of thylakoid membranes
}

\author{
Sashka Boychova Krumova • Sergey Petrovich Laptenok • \\ László Kovács • Tünde Tóth • Arie van Hoek • \\ Győző Garab · Herbert van Amerongen
}

Received: 6 February 2010/Accepted: 29 June 2010/Published online: 20 July 2010

(c) The Author(s) 2010. This article is published with open access at Springerlink.com

\begin{abstract}
We investigated the effects of digalactosyldiacylglycerol (DGDG) on the organization and thermal stability of thylakoid membranes, using wild-type Arabidopsis thaliana and the DGDG-deficient mutant, $d g d l$. Circular-dichroism measurements reveal that DGDG-deficiency hampers the formation of the chirally organized macrodomains containing the main chlorophyll $a / b$ lightharvesting complexes. The mutation also brings about changes in the overall chlorophyll fluorescence lifetimes, measured in whole leaves as well as in isolated thylakoids. As shown by time-resolved measurements, using the lipophylic fluorescence probe Merocyanine 540 (MC540), the altered lipid composition affects the packing of lipids in the thylakoid membranes but, as revealed by flash-induced electrochromic absorbance changes, the membranes retain
\end{abstract}

S. B. Krumova - S. P. Laptenok - A. van Hoek ·

H. van Amerongen

Laboratory of Biophysics, Wageningen University,

P.O. Box 8128, 6700 ET Wageningen, The Netherlands

S. B. Krumova $(\bowtie)$

Institute of Biophysics, Bulgarian Academy of Sciences, Acad.

G. Bontchev Str., bl. 21, 1113 Sofia, Bulgaria

e-mail: sashka@bio21.bas.bg

S. P. Laptenok

Department of Physics and Astronomy, Faculty of Sciences,

VU University Amsterdam, De Boelelaan 1081, $1081 \mathrm{HV}$

Amsterdam, The Netherlands

L. Kovács · T. Tóth · G. Garab

Institute of Plant Biology, Biological Research Center,

Hungarian Academy of Sciences, P.O. Box 521, 6701 Szeged,

Hungary

A. van Hoek - H. van Amerongen

MicroSpectroscopy Centre, Wageningen University,

P.O. Box 8128, 6700 ET Wageningen, The Netherlands their ability for energization. Thermal stability measurements revealed more significant differences. The disassembly of the chiral macrodomains around $55^{\circ} \mathrm{C}$, the thermal destabilization of photosystem I complex at $61^{\circ} \mathrm{C}$ as detected by green gel electrophoresis, as well as the sharp drop in the overall chlorophyll fluorescence lifetime above $45^{\circ} \mathrm{C}$ (values for the wild type-WT) occur at $4-7^{\circ} \mathrm{C}$ lower temperatures in $d g d l$. Similar differences are revealed in the temperature dependence of the lipid packing and the membrane permeability: at elevated temperatures MC540 appears to be extruded from the $d g d l$ membrane bilayer around $35^{\circ} \mathrm{C}$, whereas in WT, it remains lipid-bound up to $45^{\circ} \mathrm{C}$ and $d g d l$ and WT membranes become leaky around 35 and $45^{\circ} \mathrm{C}$, respectively. It is concluded that DGDG plays important roles in the overall organization of thylakoid membranes especially at elevated temperatures.

Keywords Arabidopsis mutants . Digalactosyl-diacylglycerol $\cdot d g d l$ mutant .

Thermal stability · Thylakoid lipids .

Thylakoid membranes

$\begin{array}{ll}\text { Abbreviations } \\ \tau_{\text {ave }} & \text { Average fluorescence lifetime } \\ \text { Chl } & \text { Chlorophyll } \\ \text { CD } & \text { Circular dichroism } \\ \text { DGDG } & \text { Digalactosyl-diacylglycerol } \\ \Delta \text { A515 } & \begin{array}{l}\text { Electrochromic absorbance change } \\ \text { FLIM }\end{array} \\ \text { Fluorescence lifetime imaging microscopy } \\ \text { LHCI } & \begin{array}{l}\text { Light-harvesting antenna complexes } \\ \text { of photosystem I } \\ \text { LHCII }\end{array} \\ & \begin{array}{l}\text { Major light-harvesting complex } \\ \text { of photosystem II }\end{array}\end{array}$

\section{Abbreviations} Average fluorescence lifetime

CD Circular dichroism

DGDG Digalactosyl-diacylglycero

$\triangle$ A515 Electrochromic absorbance change

FLIM Fluorescence lifetime imaging microscopy

LHCI Light-harvesting antenna complexes

of photosystem I

of photosystem II 
MGDG Monogalactosyl-diacylglycerol

MC540 Merocyanine 540

PSI Photosystem I

PSII Photosystem II

TCSPC Time-correlated single photon counting

WT Wild type

\section{Introduction}

Digalactosyl-diacylglycerol (DGDG) is one of the main bilayer-prone lipid species in thylakoid membranes of higher plants, constituting about $30 \%$ of the total lipid content (Douce and Joyard 1996). Its structural importance is well established for several (super)complexes of the photosynthetic machinery. It has been shown to be bound to photosystem II (PSII) (Loll et al. 2005, 2007), it forms hydrogen bonds with tyrosine in PSII (Gabashvili et al. 1998), and it is important for the binding of extrinsic proteins required for the stabilization of the oxygenevolving complex (Sakurai et al. 2007). DGDG was resolved in the crystal structure of major light-harvesting complex of photosystem II (LHCII), the major light-harvesting complex of PSII. The head groups of two DGDG molecules are simultaneously hydrogen bonded to the lumenal-surface amino acids from two adjacent LHCII trimers, functioning as a bridge (Liu et al. 2004; Yan et al. 2007). DGDG appears to be required for the formation of 2D and 3D crystals of LHCII ( $\mathrm{Nu} \beta$ berger et al. 1993). The functional significance of this lipid was studied employing a genetic approach - a mutant of Arabidopsis (Arabidopsis thaliana) was generated which lacks more than $90 \%$ of the DGDG content of the membranes ( $d g d l$, Dörmann et al. 1995). This results in a change in the chloroplast ultrastructure - the thylakoid membranes are highly curved and displaced from the central stroma area toward the envelope, the length of both grana and stroma membranes and the total length of the thylakoid membrane are increased in the mutant (Dörmann et al. 1995). This is accompanied by a decrease of the total chlorophyll ( $\mathrm{Chl}$ ) content on a fresh weight basis of about $25 \%$, in the Chl $a / b$ ratio by about $20 \%$ and a 1.7 times higher xanthophyll content (Härtel et al. 1997); however, the amount of metabolic intermediates (products of the dark reactions of photosynthesis) were found to be indistinguishable from those of the wild type (WT) (Härtel et al. 1998). Ivanov et al. (2006) have established that the DGDG deficiency has a larger effect on the structure of photosystem I (PSI) than on PSII: the relative abundance of the reaction center protein of PSII (PsbA) and the light-harvesting proteins associated with PSII (Lhcb1, Lhcb2, Lhcb3 and Lhcb5) are not changed in the mutant, whereas the reaction center proteins of PSI (PsaA and PsaB) are significantly reduced (by about 50\%) and the abundance of the PsaC, PsaL, and PsaH subunits is also substantially decreased compared to the WT (Ivanov et al. 2006). Moreover, unlike the WT, in $d g d l$ PSI has been shown to be less stable against treatment with chaotropic salts and the light-harvesting antenna complexes of PSI (LHCI) could more easily be detached from the core complex (Guo et al. 2005).

The modified protein content in $d g d l$ is accompanied by differences in various functional parameters. For example, the amount of non-photochemical quenching in $d g d l$ is increased at the expense of PSII photochemistry (Härtel et al. 1998); the linear electron transport between the two photosystems is restricted, the plastoquinol pool is more reduced, PSI has an increased capacity for cyclic electron transfer and the capacity for state transitions is reduced by $50 \%$ as compared to WT (Ivanov et al. 2006).

The emergence of these specific but nonetheless rather diverse effects of DGDG deficiency might be correlated with the multiplicity of DGDG-binding sites. However, as shown by Hendrickson et al. (2006) cold acclimation of the $d g d l$ mutant, while not affecting the lipid composition, led to the recovery of PSII and PSI photochemistry as well as the $\mathrm{CO}_{2}$ uptake capacity, and even the pigment composition became equivalent to that of WT. Based on these results, it was suggested that DGDG deficiency affected the global physical properties of the membranes, which in turn exerted specific effects in a temperature-dependent fashion. As discussed by Hendrickson et al. (2006) and can be inferred from literature data (e.g., Williams 1998; Harwood 1998; Garab et al. 2000) temperature-dependent modifications in the global properties can arise from the altered ratio of the bilayer to non-bilayer lipid contents.

The physical state of the lipid membrane, can influence a number of different global parameters of the thylakoid membrane, such as the macro-organization of the complexes, the packing of lipids, energy migration and trapping, the energization and permeability of membranesparameters which have not been studied in this mutant. In this study, we focused our attention on the role of DGDG for the overall structural organization of the thylakoid membrane and its thermal stability. Taking into account that DGDG participates in both the lipid matrix and in the protein structures, we investigate DGDG's effects on the properties of these two environments separately. Our results reveal significant alterations in the overall organization of the thylakoid membranes in $d g d l$ and decreased thermal stability of the chirally organized LHCII-containing protein macroaggregates and also of the PSI supercomplexes. These changes are accompanied by changes in the fluorescence lifetimes of chlorophyll $a$. Furthermore, the lipid packing in the thylakoid membrane appears to be 
different for the WT and $d g d l$, especially at elevated temperatures, where the energization of $d g d l$ membranes is hampered by an increased permeability.

\section{Materials and methods}

Plant material

Both the WT Arabidopsis thaliana (Arabidopsis) ecotype Columbia and the $d g d l$ mutant were grown under 16-hlight/8-h-dark cycle at $20 / 18^{\circ} \mathrm{C}$ (day/night), light intensity of $200-250 \mathrm{~W} \mathrm{~m}^{-2}$ at about $70 \%$ humidity. The plants used in the experiments were 28-35 days old.

\section{Isolation of thylakoid membranes}

Dark-adapted leaves were homogenized in a medium containing $50 \mathrm{mM}$ Tricine (pH 7.5), $400 \mathrm{mM}$ sorbitol, $5 \mathrm{mM} \mathrm{MgCl}_{2}$ and $5 \mathrm{mM} \mathrm{KCl}$; the suspension was filtered through four layers of cheese cloth and centrifuged for $4 \mathrm{~min}$ at $4,000 \times \mathrm{g}$. The chloroplasts were osmotically shocked in a hypotonic medium containing $50 \mathrm{mM}$ Tricine (pH 7.5), $5 \mathrm{mM} \mathrm{MgCl}_{2}$ and $5 \mathrm{mM} \mathrm{KCl}$, and centrifuged for $5 \mathrm{~min}$ at $6,000 \times g$. After washing in the same medium supplemented with $400 \mathrm{mM}$ sorbitol, the pellet was resuspended in this isotonic medium and used for the fluorescence and circular-dichroism measurements.

\section{Green (native) gel electrophoresis}

Isolated thylakoid membranes from WT and $d g d l$ were loaded on a polyacrylamide gel, as described in De Bianchi et al. (2008). The samples were incubated for $10 \mathrm{~min}$ at defined temperatures. Densitometry analysis was performed using Gel-pro analyser 3.1 software.

\section{Circular-dichroism measurements}

Circular dichroism (CD) was measured on isolated thylakoid membranes between 400 and $800 \mathrm{~nm}$ using a Jasco J-715 spectropolarimeter. The Chl content of the samples was adjusted to $15 \mu \mathrm{g} \mathrm{ml}^{-1}$, the optical pathlength of the cell was $1 \mathrm{~cm}$. The spectra were recorded in steps of $1 \mathrm{~nm}$ with an integration time of $2 \mathrm{~s}$, a band-pass of $2 \mathrm{~nm}$, and scanning speed of $100 \mathrm{~nm} \mathrm{~min}{ }^{-1}$. The samples were sequentially thermostated for $10 \mathrm{~min}$ at each temperature starting from $3^{\circ} \mathrm{C}$ up to $80^{\circ} \mathrm{C}$. Each experiment was repeated five times with freshly isolated thylakoids.

The amplitudes of the different CD bands were determined using reference wavelengths, e.g., by the subtraction of the maximum intensity of the positive signal at a specified wavelength and the corresponding minimum of the negative signal (for example the amplitude of the $448-459 \mathrm{~nm}$ band was obtained by subtracting the $\mathrm{CD}$ at $459 \mathrm{~nm}$ from the signal at $448 \mathrm{~nm}$ ). For strongly overlapping $\mathrm{CD}$ bands, such as the $\mathrm{CD}$ band at $685 \mathrm{~nm}$ and at $650 \mathrm{~nm}$, the amplitude was estimated by subtracting a reference zero-value $\mathrm{CD}$ signal $\left(\mathrm{CD}_{(685-730)}\right.$ and $\left.\mathrm{CD}_{(610-650)}\right)$. The transition temperature $\left(T_{\mathrm{m}}\right)$ is defined as the temperature at which the intensity of the $\mathrm{CD}$ band or band-pair is decreased by $50 \%$ of its value at $25^{\circ} \mathrm{C}$, similar to Cseh et al. (2000).

Chl $a$ time-resolved fluorescence measurements

The Chl $a$ fluorescence decay curves were measured using two techniques: (i) in vivo fluorescence lifetime imaging microscopy (FLIM) measurements on detached but intact leaves at room temperature $\left(22^{\circ} \mathrm{C}\right)$ (similar to Broess et al. 2009) and (ii) time-correlated single photon counting (TCSPC) measurements on isolated thylakoid membranes at different temperatures.

\section{Fluorescence lifetime imaging microscopy}

Fluorescence lifetime imaging microscopy (FLIM) was performed in vivo on detached leaves of WT and $d g d l$, using the setup described previously (Borst et al. 2005). In short, two-photon excitation pulses $(860 \mathrm{~nm}, 150 \mathrm{fs}$ pulse duration, $76 \mathrm{MHz}$ repetition rate) were focused into the sample with a $60 \times$ water immersion objective lens. Fluorescence was detected via non-descanned single photon counting detection, through two band-pass filters of $700 \mathrm{~nm}(75 \mathrm{~nm}$ width). Images of $64 \times 64$ pixels were obtained, with 1024 time channels of 12 ps. The fluorescence was collected for $30 \mathrm{~min}$; low excitation power (of the order of $600 \mu \mathrm{W}$ average power) was used to keep the reaction centers open and to minimize photodamage. The fluorescence decays were analyzed by software provided by Becker \& Hickl (SPCImage). All measurements were performed at $22^{\circ} \mathrm{C}$. The plants were dark-adapted at $20^{\circ} \mathrm{C}$ for $30 \mathrm{~min}$ before the measurements.

\section{Time-correlated single photon counting}

Time-correlated single photon counting (TCSPC) was used to perform time-resolved fluorescence measurements using a setup described earlier (Borst et al. 2005). For the fitting procedure, the dynamic instrumental response of the experimental setup was recorded using the fast and singleexponential fluorescence decay $(6 \mathrm{ps})$ of the reference compound pinacyanol in methanol (van Oort et al. 2008). Data analysis was performed using the computer program described earlier (Digris et al. 1999; Novikov et al. 1999). The fit quality was evaluated from $\chi^{2}$, and from the plots of 
the weighted residuals and the autocorrelation thereof (Visser et al. 2008). Typical values of $\chi^{2}$ were 1.0-1.1.

For Chl $a$ fluorescence measurements, the samples were excited at $470 \mathrm{~nm}$, and the emission was collected using an interference filter at $688 \mathrm{~nm}$ with a bandwidth of $10 \mathrm{~nm}$. The samples were sequentially thermostated at increasing discrete temperatures, between 7 and $70^{\circ} \mathrm{C}$, for $10 \mathrm{~min}$ at each temperature. The decay curves were analyzed by a four-exponential model; for each decay trace, the average lifetime $\left(\tau_{\text {ave }}\right)$ was calculated by the formula:

$\tau_{\mathrm{ave}}=\sum_{i=1}^{n} \alpha_{i} \tau_{i}$

$\tau$ being the fluorescence lifetime and $\alpha$ the preexponential factor proportional to the fractional population, with $\sum_{i=1}^{n} \alpha_{i}=1$. For the calculation of $\tau_{\text {ave }}$, the minor contribution (typically about 1-2\%) of a component with a lifetime above $1 \mathrm{~ns}$, originating from closed reaction centers, was not taken into account. The mean value of $\tau_{\text {ave }}$ and its standard error presented in this article were determined from five different decay curves measured on different samples.

Time-resolved fluorescence measurements of Merocyanine 540

For studying the lipid packing the lipophilic fluorescence probe, Merocyanine 540 (MC540, purchased from SigmaAldrich) was added, from a $1 \mathrm{mM}$ ethanol stock solution (to a final concentration of $0.2 \mu \mathrm{M}$ ), to a suspension of thylakoid membranes (containing $20 \mu \mathrm{g} \mathrm{Chl} \mathrm{ml}^{-1}$ ) and incubated for $30 \mathrm{~min}$ before the experiments. During this time, the sample was gently stirred and kept on ice in the dark. Longer incubation with MC540 did not result in increased incorporation of the probe (see Krumova et al. 2008a and references therein).

For fluorescence lifetime measurements, the TCSPC setup described in the previous section was used. The excitation wavelength was set to $570 \mathrm{~nm}$, and the emission was collected between 610 and $630 \mathrm{~nm}$ using a Schott OG $610 \mathrm{~nm}(3 \mathrm{~mm})$ cut-off filter and a Balzers K60 interference filter. During the measurements the samples were sequentially thermostated at increasing discrete temperatures, between 7 and $45^{\circ} \mathrm{C}$, for $15 \mathrm{~min}$ at each temperature. The MC540 measurements, as in the more systematic study using the same lipid probe in isolated thylakoids (Krumova et al. 2008a), are confined to this temperature interval with no protein degradation (Dobrikova et al. 2003) but significant changes in the lipid packing (detected also by

${ }^{31} \mathrm{P}-\mathrm{NMR}$, Krumova et al. 2008b); above $45^{\circ} \mathrm{C}$, thylakoid lipids segregate in large quantities from the membrane and form extended non-bilayer structures (Gounaris et al. 1984).
For the analysis of the fluorescence decay, the threeexponential model introduced earlier (Krumova et al. 2008a) was used, which assumes a partition of MC540 between the aqueous phase with short ( $<200 \mathrm{ps})$ lifetime and the two lipid phases with $\sim 1$ - and $\sim 2$-ns lifetimes.

Since these three types of microenvironments are the same for WT and $d g d l$, the MC540 fluorescence lifetimes for the five different WT or $d g d l$ samples were linked during the fitting procedure (resulting, at a given temperature, in equal lifetime values for both samples) whereas their relative amplitudes were left free. In this way, the changes in the distribution of MC540 over the different environments can be followed for WT and $d g d l$.

Electrochromic absorbance transients

Electrochromic absorbance changes $(\Delta \mathrm{A} 515)$, induced by saturating single turnover flashes, were measured at $515 \mathrm{~nm}$ on detached leaves, in a setup described earlier (Büchel and Garab 1995). The plants used for the measurements were dark-adapted at $20^{\circ} \mathrm{C}$ for $30 \mathrm{~min}$, and detached leaves of WT and $d g d l$ were infiltrated with water, incubated for $10 \mathrm{~min}$ at different temperatures, and then measured at $25^{\circ} \mathrm{C} ; 64$ kinetic traces were collected with a repetition rate of $1 \mathrm{~s}^{-1}$ and averaged; the duration of the flashes was about $5 \mu \mathrm{s}$; the time constant of the measurements was adjusted to $100 \mu \mathrm{s}$. The measurements were repeated five times with leaves from different plants.

\section{Results}

Pigment-protein complexes: (macro-)organization, excitation energy migration, and trapping

\section{Circular dichroism}

The CD spectra of thylakoid membranes isolated from WT and $d g d l$ are presented in Fig. 1a. It can be seen that at $25^{\circ} \mathrm{C}$, the amplitudes of the $(-) 650 \mathrm{~nm}$ band, arising from excitonic interactions of $\mathrm{Chl} b$ in monomeric and trimeric LHCII, were approximately identical in WT and $d g d l$. Also, the Chl $a$ CD signals between 400 and $450 \mathrm{~nm}$ were not affected significantly by the deficiency of DGDG. In contrast, the intensities of the main $\Psi$-type $C D$ bands, between 660 and $700 \mathrm{~nm}$ and at around $505 \mathrm{~nm}$, were substantially smaller for $d g d l$ (Fig. 1a). (For the origin of the main $\mathrm{CD}$ bands in thylakoids, see, e.g., Garab and van Amerongen 2009).

The thermal destabilization of different protein complexes was monitored via the amplitudes of their corresponding CD bands. The (-)650 nm band exhibited the same temperature dependence for WT and $d g d l$ and 

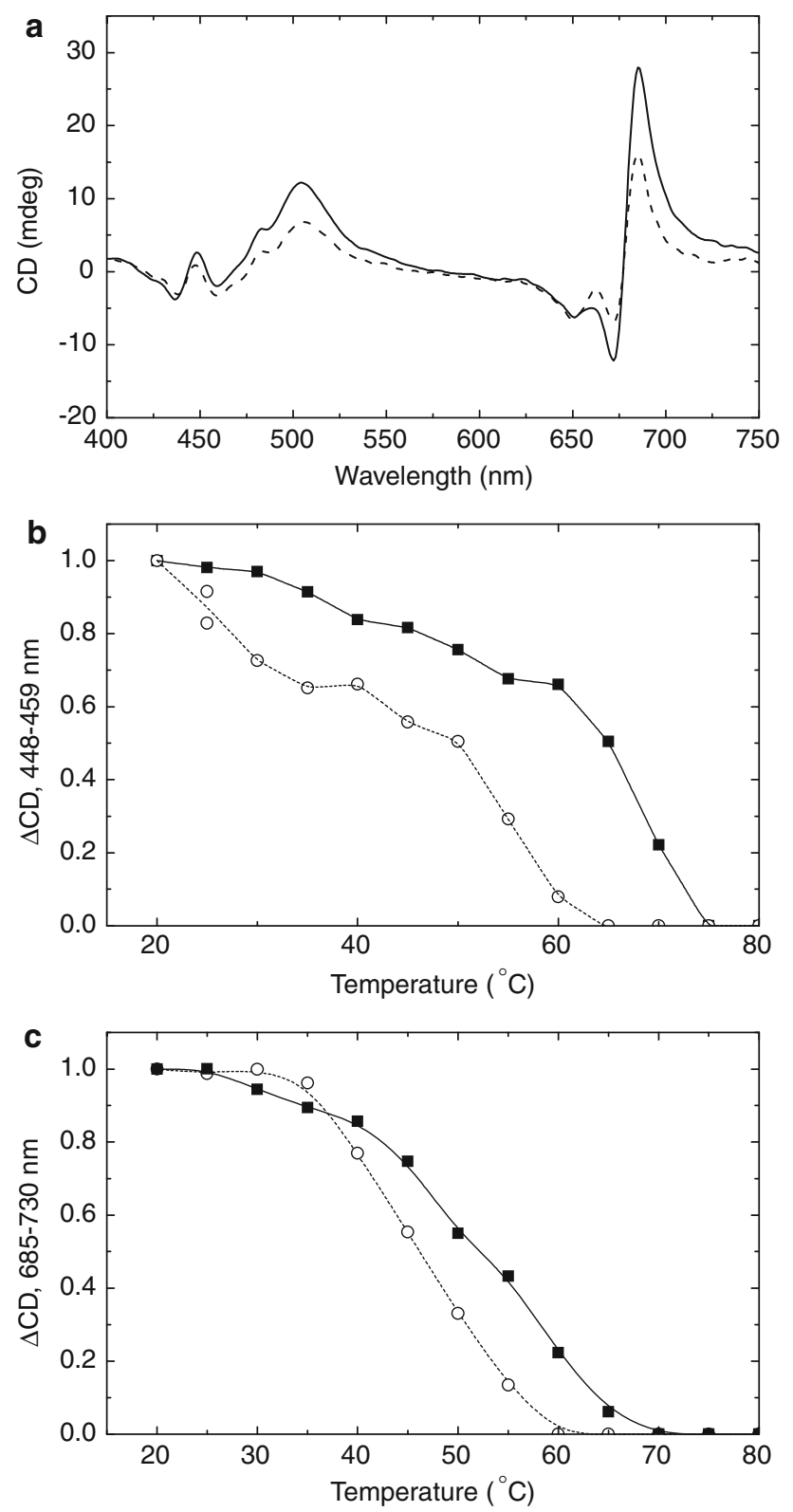

Fig. 1 a Typical CD spectra of thylakoid membranes isolated from WT (solid line) and dgdl (dashed line) leaves. The spectra were measured at $25^{\circ} \mathrm{C}$ at identical $\mathrm{Chl}$ concentrations $\left(15 \mu \mathrm{g} \mathrm{ml}{ }^{-1}\right)$. Typical temperature dependence of the $448-459 \mathrm{~nm}$ (b) and 685-730 nm (c) CD signals for the WT (filled square) and dgd1 (open circle). The lines (solid for the WT and dashed for dgdl) serve as a guide to the eye. The experiments were repeated five times and resulted in very similar differences in the $\mathrm{CD}$ spectra and their thermal behavior

displayed essentially identical transition temperatures $\left(T_{\mathrm{m}}\right)$ at $\sim 60^{\circ} \mathrm{C}$ (Table 1 ). On the other hand, the mutation substantially affected the thermal stability of the $\mathrm{Chl}$ $a$ excitonic bands at around $450 \mathrm{~nm}$, determined either as $\mathrm{CD}_{(448-438)}$ (not shown) or $\mathrm{CD}_{(448-459)}$ (Fig. 1b). The $T_{\mathrm{m}}$ values were lower by $\sim 6^{\circ} \mathrm{C}$ for the mutant than for the WT (Table 1). The $\Psi$-type signal $\left(\mathrm{CD}_{(685-730)}\right)$ also exhibited different temperature dependencies for $\mathrm{WT}$ and $d g d l$ (Fig. 1c). The transition temperature for this band was $54 \pm 2^{\circ} \mathrm{C}$ for the WT, whereas for $d g d l$ it was found at $48 \pm 1^{\circ} \mathrm{C}$ (Table 1$)$.

\section{Green (native) gel electrophoresis}

In order to discriminate between the thermal behavior of the different photosynthetic complexes, green gel electrophoresis of heat-treated thylakoid membranes from WT and $d g d l$ was performed (Fig. 2a) and analyzed for the contents of PSI supercomplexes (Fig. 2b) and LHCII trimers (Fig. 2c). The data show that the PSI supercomplex in $d g d l$ is less stable upon heat treatment than the WT-the intensity of the corresponding green gel band decreases by $50 \%$ at $57^{\circ} \mathrm{C}$ for $d g d 1$ and at $61^{\circ} \mathrm{C}$ for WT, respectively (Fig. 2b). In contrast, the destabilization of LHCII trimers follows the same pattern in both the WT and $d g d l$ up to $65^{\circ} \mathrm{C}$ (Fig. 2c).

\section{Chlorophyll a fluorescence lifetime}

The functional activity of the photosystems was studied with the aid of Chl $a$ fluorescence lifetime measurements, using microscopic (FLIM) and macroscopic (TCSPC) measurements.

The FLIM images are plotted in Fig. 3a, b (WT) and c, d ( $d g d l)$. The recorded fluorescence originates from Chls in the chloroplasts. Thus, the bright spots in the intensity images (Fig. 3a, c) originate from distinct chloroplasts. Their shape is not well defined in the FLIM images due to the fact that the brightness of the individual organelles is proportional to the intensity of the fluorescence emission.

Table 1 Transition temperatures $\left(T_{\mathrm{m}}\right)$ of selected CD bands or band pairs for WT and dgdl thylakoid membranes

\begin{tabular}{llll}
\hline $\begin{array}{l}\text { CD signal } \\
(\mathrm{nm})\end{array}$ & Assignment & $\begin{array}{l}T_{\mathrm{m}^{\prime}}{ }^{\circ} \mathrm{C} \\
(\mathrm{WT})\end{array}$ & $\begin{array}{l}T_{\mathrm{m}^{\prime}}{ }^{\circ} \mathrm{C} \\
(d g d l)\end{array}$ \\
\hline $685-730$ & $\Psi$-type & $54 \pm 2$ & $48 \pm 1$ \\
$685-671$ & $\Psi$-type & $54 \pm 1$ & $49 \pm 1$ \\
$505-550$ & $\Psi$-type & $56 \pm 1$ & $51 \pm 1$ \\
$610-650$ & Excitonic $(\mathrm{Chl} b$, & $61 \pm 2$ & $58 \pm 2$ \\
& LHCII) & $59 \pm 2$ & $54 \pm 1$ \\
$448-459$ & Excitonic $(\mathrm{Chl} a)$ & $57 \pm 1$ & $50 \pm 1$ \\
$448-438$ & Excitonic $(\mathrm{Chl} a)$ & 57 \\
\hline
\end{tabular}

The membranes were thermostated for $10 \mathrm{~min}$ at different temperatures in the range between 5 and $80^{\circ} \mathrm{C}$ before recording the $\mathrm{CD}$ spectra at the given temperature; the amplitudes for the individual bands were calculated from the difference in the intensity at specific wavelengths (see also the text). $T_{\mathrm{m}}$ is defined as the temperature at which the intensity of the CD band is decreased to $50 \%$ of its value at $25^{\circ} \mathrm{C}$. The values for $T_{\mathrm{m}}$ and their standard errors are determined from five independent experiments 
Fig. 2 a Native green gel analysis of heat-treated WT and dgd1 thylakoid membranes at different temperatures. The samples are treated for $10 \mathrm{~min}$ before loading on the gel. The main bands denoted as I and II represent PSI supercomplex and LHCII trimers, respectively. The temperature dependencies of the stability of PSI supercomplex and LHCII trimer determined from the green-gel density profiles of heat treated WT (filled square) and $d g d 1$ (open square) thylakoid membranes are presented in (b) and (c), respectively. The samples were treated for $10 \mathrm{~min}$ at the specified temperatures before loading on the gel
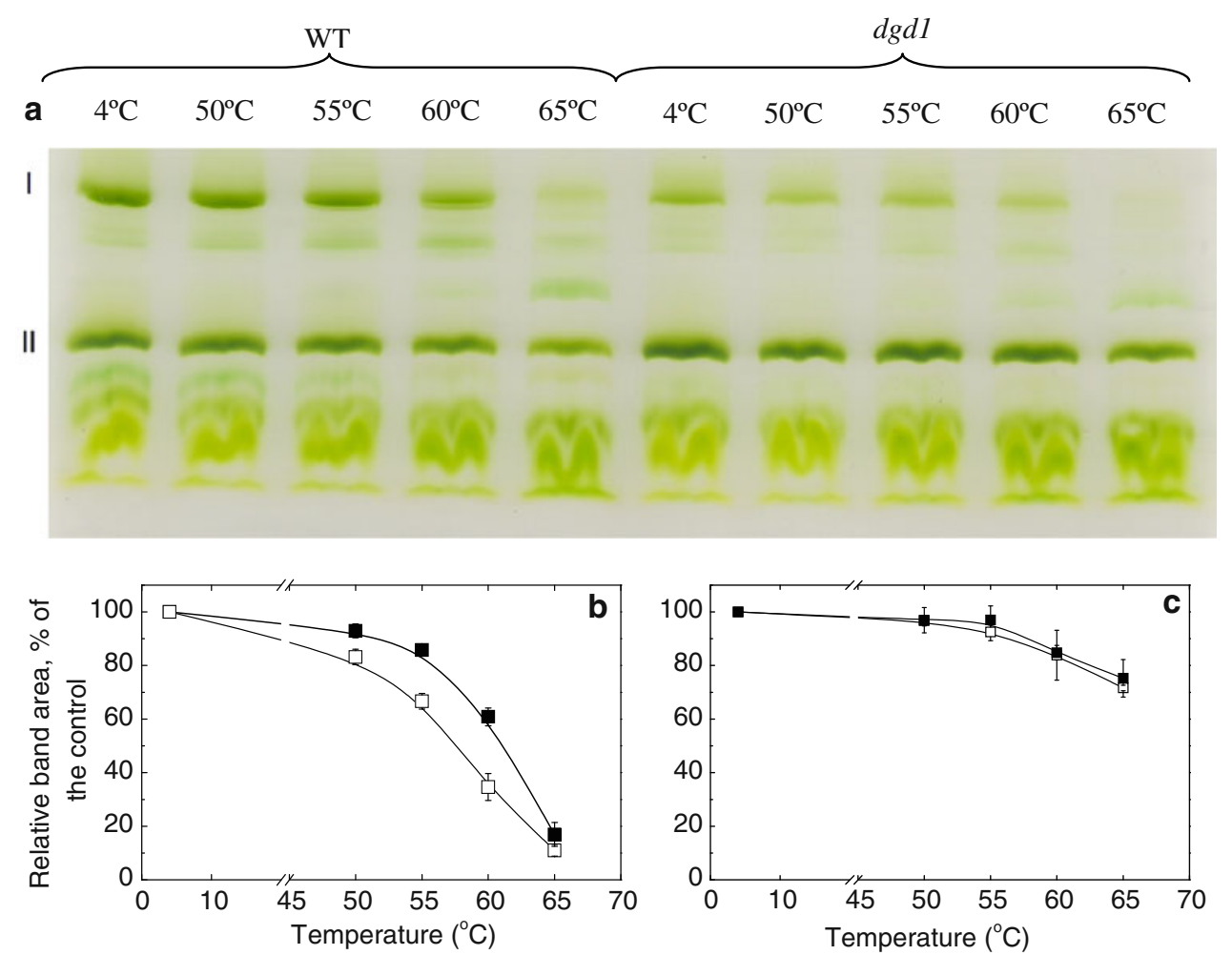

Therefore, the chloroplasts being located in the focal plane are observed as bright objects, whereas the lower intensity pixels probably represent somewhat out-of-focus chloroplasts. The fluorescence decay traces recorded for each pixel were analyzed by a three-exponential model from which an average lifetime per pixel was calculated. These average lifetimes are plotted in Fig. $3 b$ and $d$ for the WT and $d g d 1$, respectively. The sum of the decay curves recorded for all the pixels in the image of WT and $d g d l$ leaves is presented in Fig. 3e. The distribution histogram of the average lifetime is presented in Fig. 3f, which also clearly shows that it is longer for the mutant-the average fluorescence lifetime in the majority of the pixels of the WT-image is $180-220 \mathrm{ps}$, whereas for the $d g d l$-image it is about 250-300 ps.

The FLIM setup used can only be applied for measurements at $22^{\circ} \mathrm{C}$. In order to check the temperature dependence of the average $\mathrm{Chl} a$ fluorescence lifetime $\left(\tau_{\text {ave }}\right)$, it was determined for isolated intact thylakoid membranes using the TCSPC technique. The fluorescence decay curves for WT and $d g d l$ are shown in Fig. 4a and the parameters obtained from the fit are plotted as a table in the figure. At $25^{\circ} \mathrm{C}$, the fitting analysis results in longer fluorescence lifetimes for $d g d l$ than for $\mathrm{WT}-\tau_{\mathrm{ave}}=$ $202 \pm 5$ ps for WT and $236 \pm 13$ ps for $d g d l$ (Fig. 4b); these values are similar to the ones determined using the FLIM technique (Fig. 3e). It must be noted that the temperature dependence of the average fluorescence lifetime is characterized by different phases (Fig. 4b); interestingly, these variations were less marked for $d g d l$ than for the WT. Interpretation of these results is beyond the scope of this study. The only aspect of the temperature dependence that we want to point out is the strong decrease of the average lifetime above $50^{\circ} \mathrm{C}$ (reaching $83 \mathrm{ps}$ at $65^{\circ} \mathrm{C}$ ). For $d g d 1$ the same sharp drop in $\tau_{\text {ave }}$ occurs at lower temperatures and begins at around $45^{\circ} \mathrm{C}$ (Fig. 4b).

\section{Lipid matrix: lipid packing and membrane permeability}

In order to study the global physical properties of the lipid matrix of thylakoids, two methods were applied: (i) timeresolved fluorescence of MC540 in thylakoid membranes, which reports on the packing of the lipid molecules; and (ii) electrochromic absorbance transients on whole leaves, which probe the energization and the permeability of thylakoid membranes.

\section{Partition of MC540 in thylakoid membranes}

Using the three-exponential model for the analysis of the fluorescence decay of MC540 (see also "Materials and methods"), lifetimes of $0.19-0.23$ ns (Fig. 5a), 0.66-1.08 ns (Fig. 5b), and 1.71-2.15 ns (Fig. 5c) were obtained; the lifetimes shorten with the increase of 

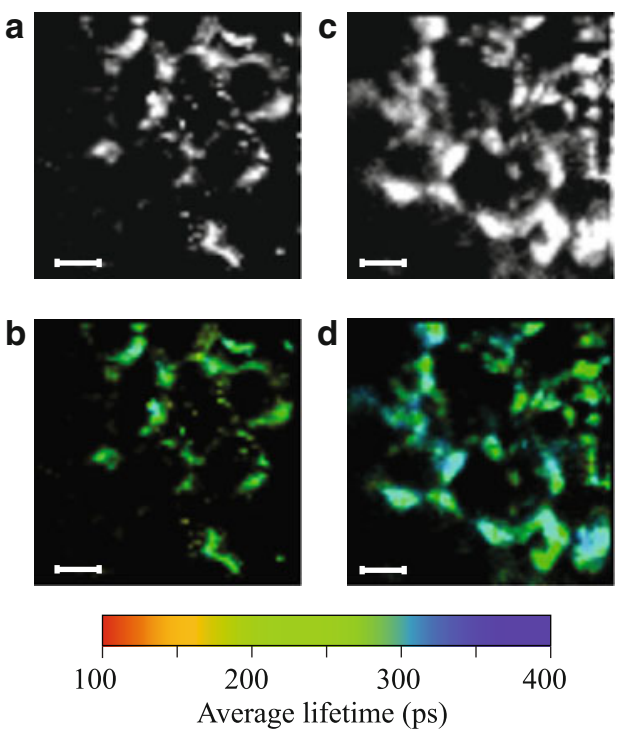

Fig. 3 FLIM results on dark-adapted detached WT and $d g d l$ leaves. The fluorescence images are shown in panel (a) for the WT, and panel (c) for $d g d 1$. The color-coded average fluorescence lifetime images are presented in panel (b) for the WT and panel (d) for $d g d 1$. Scale bars, $20 \mu \mathrm{m}$. The decay traces recorded for each pixel in the images were added, and their sums are presented in panel (e) for the WT

temperature. In this article, they are referred to as 200-ps, 1-ns, and 2-ns components, respectively.

As shown in Fig. $5 \mathrm{a}-\mathrm{c}$, the relative amplitudes of the different lifetime components of MC540 differ for WT and $d g d l$. The relative amplitude of the $200 \mathrm{ps}$ component remains constant up to $45^{\circ} \mathrm{C}$ for WT, whereas for $d g d l$ it increases with the increase of temperature, evidently at the expense of the 1-ns component. This appears to occur especially above $25-30^{\circ} \mathrm{C}$ (Fig. 5a). A comparison of the relative amplitudes of the 1- and 2-ns components in $d g d l$ and WT) reveals that for WT the relative amplitude of the 2-ns component is slightly larger than that of the 1-ns component, indicating that the amounts of MC540 incorporated into the bilayer and located on the surface are almost equal (Fig. 5b, c). In contrast, for $d g d l$, the relative amplitude of the 1-ns component is significantly larger than that of the 2-ns component (Fig. 5b, c).

If the two slow components originate from a broad distribution of lifetimes (cf. Krumova et al. 2008a), then their weighted average lifetime is a more appropriate parameter to consider. As can be seen in Fig. 5 d, at $7{ }^{\circ} \mathrm{C}$ this average lifetime is shorter for $d g d l(1.35 \pm 0.1 \mathrm{~ns})$ than for WT $(1.52 \pm 0.01 \mathrm{~ns})$. The average lifetime for both WT and $d g d l$ is decreasing with the increase of temperature, but the average lifetime of $d g d l$ remains shorter at all temperatures between 7 and $35^{\circ} \mathrm{C}$; at $45^{\circ} \mathrm{C}$ the two lifetimes become almost identical, about $1.1 \mathrm{~ns}$.
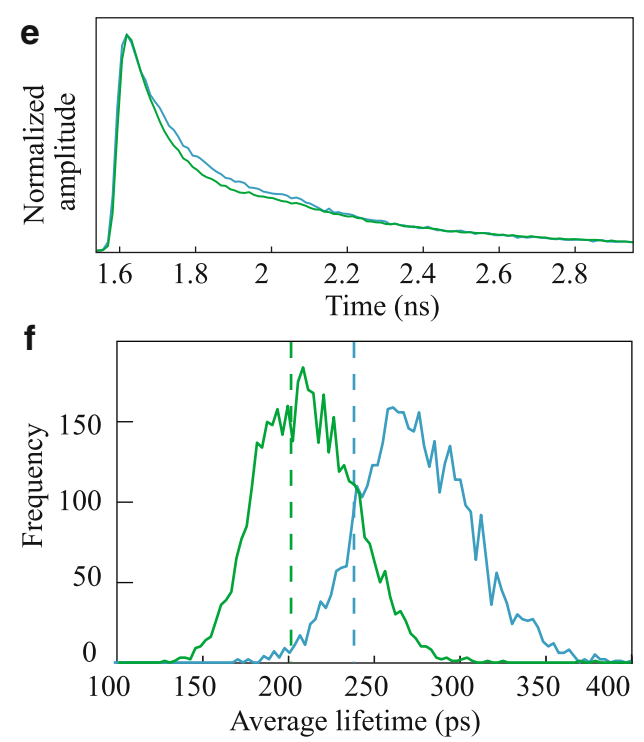

(green trace) and dgdl (blue trace). The histograms of the average lifetimes, obtained from a total of 4,096 pixels for each sample, and plotted with 3 ps steps, are given in panel (f) (green curve for the WT and blue for $d g d 1$ ). The dashed lines represent the average lifetime values for WT and $d g d l$, obtained for isolated thylakoid membranes by TCSPC at $25^{\circ} \mathrm{C}$

\section{Electrochromic absorbance changes (4A515) in WT and $d g d 1$}

In order to test the membrane permeability, electrochromic absorbance change $(\triangle \mathrm{A} 515)$ measurements were performed.

On the time scale of the experiment, the rise of $\Delta \mathrm{A} 515$, due to primary charge separations, is instantaneous. The initial amplitude of $\Delta \mathrm{A} 515$ (for samples with identical Chl concentration) differs for WT and $d g d l$, as can be seen in Fig. $6 \mathrm{a}$ and b. At $25^{\circ} \mathrm{C}$, the decay time of $\Delta \mathrm{A} 515$ for the mutant $\left(t_{1 / 2}=226 \pm 15 \mathrm{~ms}\right)$ is essentially the same as for the WT $\left(t_{1 / 2}=227 \pm 19 \mathrm{~ms}\right)$. For the $35^{\circ} \mathrm{C}$-treated sample, the decay of $\Delta \mathrm{A} 515$ is significantly faster for the $d g d l$ mutant (Fig. 6b); the corresponding halftimes are $237 \pm 16 \mathrm{~ms}$ for WT and $154 \pm 19 \mathrm{~ms}$ for $d g d l$. No change in the decay rate was observed for the WT leaves exposed to the same temperature; only at $40^{\circ} \mathrm{C}$, the decay becomes faster $\left(t_{1 / 2}=36 \pm 12 \mathrm{~ms}\right)$ for WT; at this latter temperature no $\Delta \mathrm{A} 515$ signal can be discerned for $d g d l$.

\section{Discussion}

In this article, we investigated the role of one of the major thylakoid lipids, DGDG on the global organization and thermal stability of the membranes. To this end, we used the Arabidopsis lipid mutant $d g d l$, with substantially decreased DGDG content (Dörmann et al. 1995), and measured 

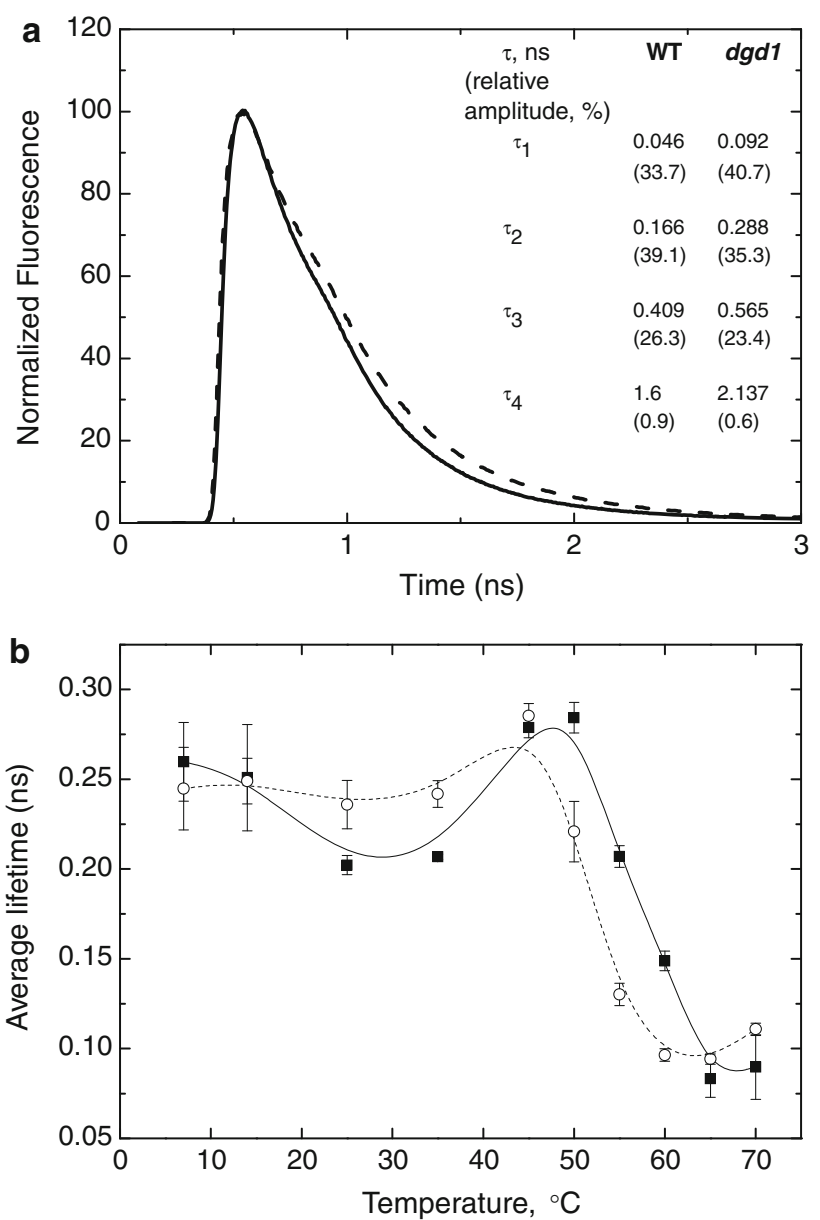

Fig. 4 a Chlorophyll a fluorescence decay traces for isolated thylakoid membranes from WT (thick line) and dgdl (dashed line), recorded by TCSPC. The presented curves are the sums of five independent measurements on different preparations. The excitation wavelength is $430 \mathrm{~nm}$, and the emission is recorded at $688 \mathrm{~nm}$ at $25^{\circ} \mathrm{C}$. The corresponding fits (fluorescence lifetimes $(\tau)$ and relative amplitudes, given in brackets) are also presented. b Temperature dependence of the average fluorescence lifetime for the WT (filled square) and $d g d l$ (open circle). Details about the fitting procedure are described in "Materials and methods". The lines (solid for WT and dashed for $d g d 1)$ serve as a guide to the eye. The average lifetime values and their standard errors are determined from five independent experiments

different physical parameters, such as $\mathrm{CD}$ of the thylakoid membranes $\mathrm{Chl}$ a fluorescence lifetimes and the fluorescence lifetime distribution of the lipophylic dye MC540 as well as the electrochromic absorbance changes along with their temperature-dependencies in WT and $d g d l$ samples.

Effects of DGDG on the global organization of thylakoid membranes

Dörmann et al. (1995) have revealed major ultrastructural differences in the organization of the thylakoid membranes between the $d g d l$ and the WT such as increased number of thylakoids per granum and longer granal and stromal thylakoids. It is well known that the stacking of thylakoids and the lateral macro-organization of the pigment-protein complexes in the membrane are interrelated (reviewed by Mustárdy and Garab 2003; Dekker and Boekema 2005) but $d g d l$ is poorly characterized in this respect.

In order to obtain information on the global organization of pigment-protein complexes in $d g d l$ thylakoid membranes, we performed CD spectroscopic measurements. We also performed $\mathrm{Chl}$ fluorescence lifetime measurements to provide an insight into the energy migration and trapping capabilities of the membranes in relation to the altered composition of the membranes and the macro-organization of the complexes. The effect of DGDG deficiency on the packing of lipids and the energization of membranes were tested with the aid of MC540 fluorescence lifetime measurements and by measuring electrochromic absorbance transients.

Circular-dichroism (CD) spectroscopy in the visible range is a valuable tool for probing the molecular architecture of the complexes and supercomplexes and their macro-organization in the membrane system (Garab and van Amerongen 2009). Two types of CD bands are relevant for the study of thylakoid membranes described a follows:

(i) Excitonic bands which originate from short-range (nanometer scale) excitonic interactions between pigments within a pigment-protein complex or on adjacent complexes (Tinoco 1962; De Voe 1965; Somsen et al. 1996; Garab and van Amerongen 2009), and can be used for testing the intactness of individual complexes or supercomplexes. Such interactions give rise to conservative band structures-i.e., the positive and negative bands of the split spectrum have equal areas. In a system as complex as the thylakoid membrane, a variety of excitonic bands is superimposed on top of each other. These are difficult to discriminate, and here, we shall use only two characteristic bands, at around 650 and $440 \mathrm{~nm}$. It has been established that the $(-) 650 \mathrm{~nm}$ band originates from $\mathrm{Chl} b$ and is regarded as a fingerprint of the LHCII complexes (van Metter 1977; Georgakopoulou et al. 2007), while the CD bands that appear between 400 and $450 \mathrm{~nm}$ mainly originate from Chl $a$ (Garab et al. 1991). The intensity of the $(-) 650 \mathrm{~nm}$ CD band remains unchanged in $d g d l$, which demonstrates that the molecular architecture of LHCII is not significantly affected by the mutation.

Fig. 5 Temperature dependencies of the parameters, obtained after the analysis of the fluorescence decays recorded for MC540 in WT and $d g d l$ thylakoid membranes. a-c Lifetime components (blue symbols) and their respective amplitudes in WT (full black symbols) and $d g d 1$ (open black symbols). d Weighted average lifetimes of the two long-lived components for WT (filled circle) and dgdl (open circle). The samples were thermostated for $10 \mathrm{~min}$ at each temperature before starting the measurements. For further details for the fitting model see also "Materials and methods" and text 

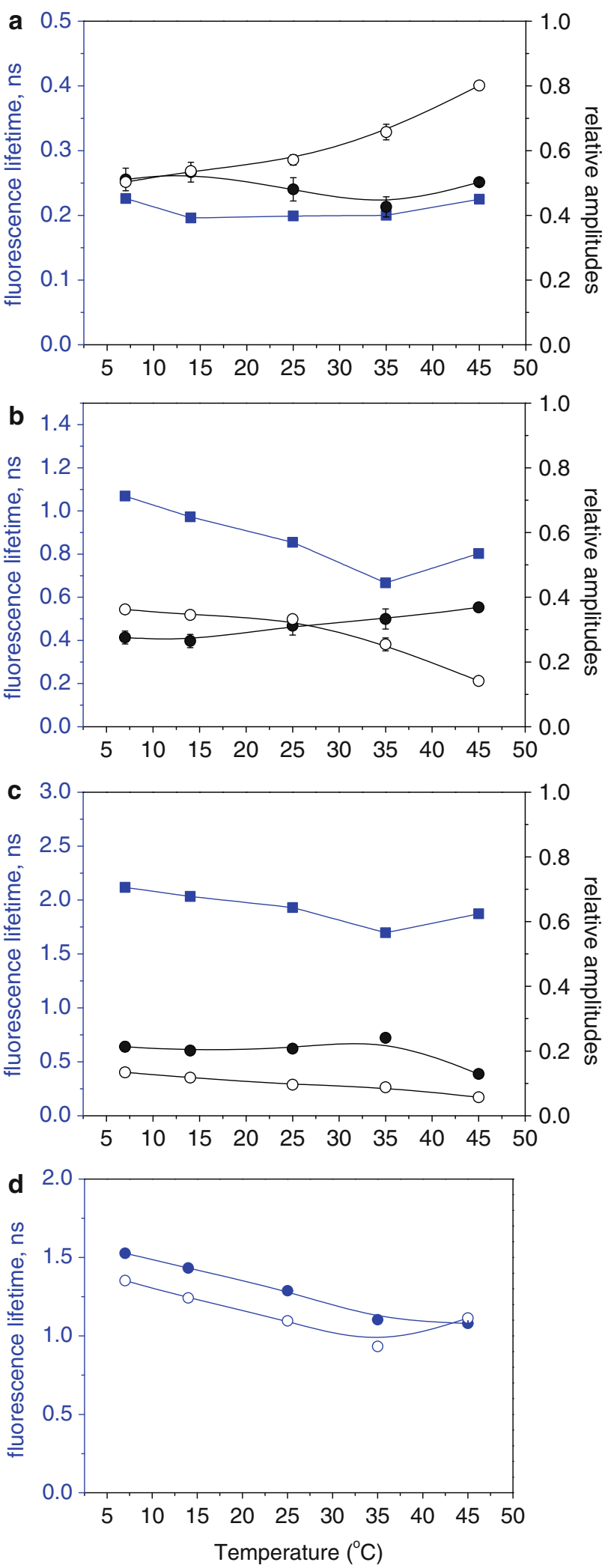

(ii) $\Psi$-type CD bands-high-intensity bands, originating from long-range order (hundreds of nanometers) of the chromophores in chirally-organized macroarrays. They are
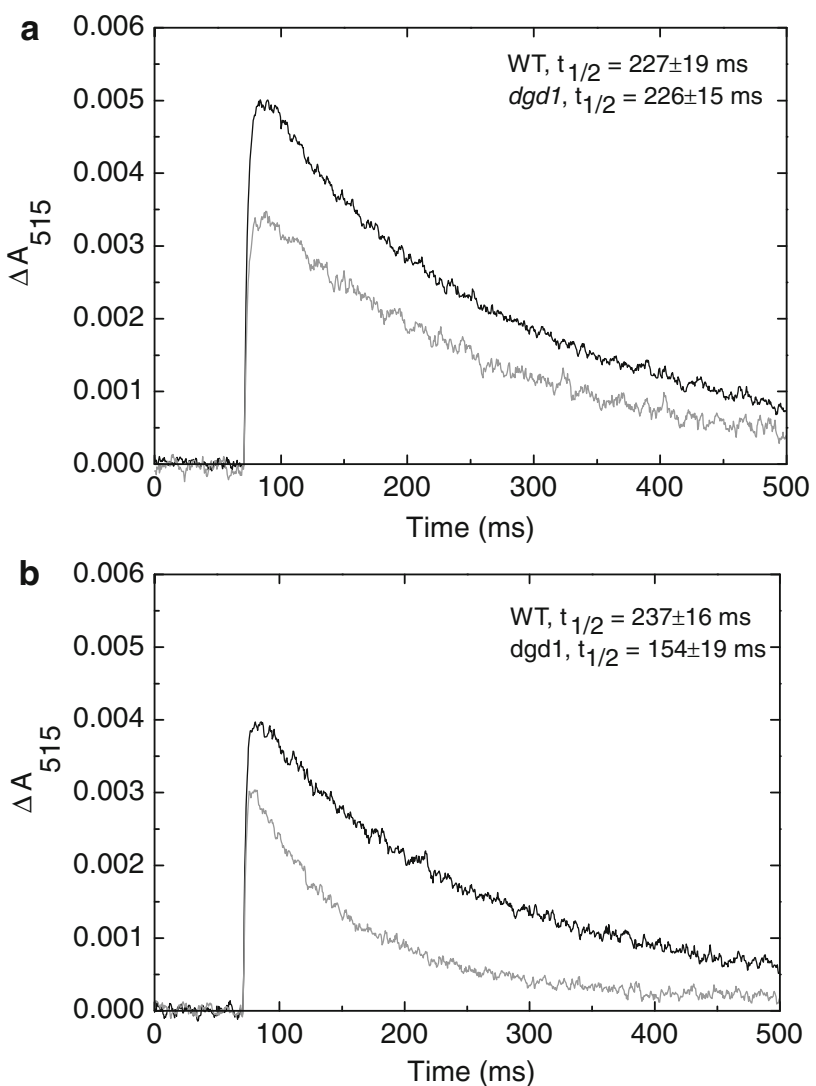

Fig. 6 Typical electrochromic absorbance transients recorded at $515 \mathrm{~nm}(\triangle \mathrm{A} 515)$, induced by saturating single-turnover flashes on detached WT (black trace) and dgdl mutant (gray trace) leaves incubated in the dark for $10 \mathrm{~min}$ at $25^{\circ} \mathrm{C}$ (a) and $35^{\circ} \mathrm{C}$ (b) and subsequently measured at $25^{\circ} \mathrm{C}$. The kinetic traces are obtained by averaging 64 transients with a repetition rate of $1 \mathrm{~s}^{-1}$. The corresponding decay halftimes for WT and $d g d l$ (average from five independent experiments and their corresponding standard errors) are also plotted in the figure

very intense and "anomalously shaped" (Garab 1996); in thylakoids and isolated LHCII-aggregates they appear at around (+)685, (-)671 nm and (+)505 nm (Barzda et al. 1994; Dobrikova et al. 2003); these bands are also associated with long tails outside the principal absorbance bands, which originate from differential scattering of the left and right circularly polarized light (Garab 1996). $\Psi$-type bands correlate with the macro-organization of the main $\mathrm{Chl} a / b$ light harvesting complexes, e.g., in LHCII-only domains, as indicated by correlations between the intensity of these bands and the LHCII-content of the sample (e.g., Garab et al. 1991; Garab and Mustárdy 1999). The arrays of PSII-supercomplexes might also contribute to the $\Psi$-type CD signal. For example, in a mutant lacking one of the minor lightharvesting complexes, namely, CP24, the macro-organization of the PSII-supercomplexes is modified as compared to WT. This results in the loss of the main $\Psi$-type band in the red at around (+)690 nm (Kovács et al. 2006). 
The intensities of the $\Psi$-type CD bands between 660 and 700 (Fig. 1a) differ for WT and $d g d l$ thylakoids. These CD signals are shown to be determined by the long-range organization of the pigment-protein complexes, in particular LHCII (e.g., Garab et al. 1991; Garab and Mustárdy 1999) and PSII-supercomplexes (Kovács et al. 2006). Thus, the reduced intensity of the main $\Psi$-type $C D$ bands $\left(\mathrm{CD}_{(685-703)}\right.$ and $\left.\mathrm{CD}_{(685-671)}\right)$ in the mutant (Fig. 1a) might either be due to a smaller size of the chiral macrodomains or to a different organization of the complexes affecting the pigment-pigment interactions. It should be noted that DGDG has been found to be required for the formation of ordered 3D crystals of LHCII (Nu $\beta$ berger et al. 1993). Hence, our CD data strongly suggest that also in vivo in the thylakoid membranes DGDG modulates the macroorganization of the main light-harvesting complexes of PSII.

As shown by Chl fluorescence lifetime measurements, alterations in the macroorganization in $d g d l$ affected only marginally the energy migration and trapping (Figs. 3, 4). The mutant exhibited a somewhat longer average $\mathrm{Chl}$ $a$ fluorescence lifetime (Figs. 3f, 4).

The assignment of the fluorescence lifetimes to particular protein complexes or macroassemblies is a rather complicated task for intact chloroplasts and isolated thylakoids, where a large variety of complexes and supercomplexes co-exist. For example, most studies on whole chloroplasts and intact thylakoid membranes suggested average values for the trapping time in PSII between $\sim 300$ and $\sim 500$ ps (e.g., Roelofs et al. 1992; Gilmore et al. 1996; Vasile'v et al. 1998). A very detailed study of the fluorescence kinetics of thylakoid membranes with varying composition was recently performed, using different combinations of excitation and detection wavelengths to assign the various lifetimes to PSI and PSII but this is not a trivial task (van Oort et al. 2010). For isolated BBY particles (containing only the granal thylakoid membranes) two major lifetimes of about 74-80 and 175-212 ps were observed (Broess et al. 2006, 2008), whereas three major decay times are found for PSI: 5-20, 20-60, 80-130 ps (Turconi et al. 1994; Croce et al. 2000; Ihalainen et al. 2002, 2005; van Oort et al. 2008; Slavov et al. 2008). Because of the various complications, only the average lifetimes $\left(\tau_{\text {ave }}\right)$ measured for WT and $d g d l$ thylakoid membranes and intact leaves are compared in this article.

The longer lifetime in $d g d l$ can most easily be explained by taking into account the lower PSI content of the membranes (Ivanov et al. 2006)—-this photosystem exhibits short lifetimes (e.g., van Oort et al. 2010). Further, excess amounts of LHCI might also contribute to the longer lifetimes-according to Ivanov et al. (2006) the amount of LHCI was unchanged; hence, a fraction of these antenna complexes might not be connected to the reaction center.
As reported by the lipophilic fluorescence probe MC540, alterations in the lipid composition in $d g d l$ bring about changes in the lipid packing. The spectroscopic properties of MC540 are determined by the dielectric constant of its local environment (Lelkes and Miller 1980). Thus, it exhibits different fluorescent lifetimes when present in different environments (interacting with lipids or solubilized in the aqueous phase). Earlier it has been shown that the shortest lifetime (200 ps) component originates from dyes in aqueous environment and the 1- and 2-ns components from MC540 in hydrophobic environments, i.e., in the lipid phase (Krumova et al. 2008a). These lifetimes might be assigned either to two discrete populations of the molecules, reflecting two different microenvironments or to a broad distribution of lifetimes due to incorporation of MC540 in a variety of environments with small differences in their physical properties.

Our data reveal significant differences in the lipid packing between $d g d l$ and WT membranes. Most prominently, the increased amplitude of the 200 ps component suggest that in $d g d l$ the MC540 molecules are more exposed to the aqueous phase than in WT (Fig. 5). The lower extent of incorporation of MC540 in the thylakoid membranes isolated from $d g d l$ in comparison with WT membranes might be due to two factors: (i) tighter lipid packing in $d g d l$, which could be the consequence of modified lipid-protein interactions and changes in the macroorganization, and/or (ii) modified surface charge of the membrane, i.e., due to conformational changes in the protein complexes or to differences in lipid-protein interactions.

Despite the altered lipid composition (increased nonbilayer:bilayer lipid ratio) and alterations in the lipid packing, the $\Delta \mathrm{A} 515$ measurements indicate that the $d g d l$ thylakoid membranes are perfectly adjusted to generate and maintain the transmembrane electrochemical potential difference at $25^{\circ} \mathrm{C}$ (Fig. 6a). $\triangle \mathrm{A} 515$ is a voltmeter of thylakoid membranes. Light-induced primary charge separation in the reaction centers, followed by vectorial transport of charges, generate a transmembrane electrochemical potential difference, which consists of a $\mathrm{pH}$ gradient and an electric potential difference. By a reverse flow of protons, the electrochemically stored energy is used for ATP synthesis (Mitchell 1966). The potential gradient can also be dissipated by the basal ion efflux, which depends on the electrical permeability of the membranes. The rise and decay of the transmembrane electrical difference can be followed by the electrochromic absorbance changes $(\triangle \mathrm{A} 515)$ of the pigments embedded in the membrane, which correlates with the transmembrane electric field (Junge 1977; Witt 1979).

We have obtained $\Delta$ A515 decay times comparable with those observed for barely under similar conditions (Garab 
et al. 1983). The initial amplitude of $\Delta \mathrm{A} 515$ is lower for dgdl than for WT, but this can be attributed to the decreased content of PSI reaction centers in the mutant (Ivanov et al. 2006). These data are also in line with the data of Härtel et al. (1997) showing that $d g d l$ is capable of maintaining a low lumenal $\mathrm{pH}$, needed for the xanthophyll cycle operation.

Effects of DGDG on the thermal stability of thylakoid membranes

The temperature dependencies of the various CD bands reveal that whereas LHCII (characterized by $(-) 650 \mathrm{~nm}$ Chl $b$ excitonic band) preserved its stability, the $\Psi$-type $\left(\mathrm{CD}_{(685-730)}\right.$ and $\left.\mathrm{CD}_{(685-671)}\right)$ and the excitonic Chl $a \mathrm{CD}$ bands $\left(\mathrm{CD}_{(448-459)}\right.$ and $\left.\mathrm{CD}_{(448-438)}\right)$ are significantly less stable in the mutant (Fig. 1; Table 1). The latter two Chl $a$ CD signals most probably originate from the core complexes of PSII and/or PSI which bind only Chl $a$ (Chitnis 2001; Smith et al. 2002; Ben-Shem et al. 2003), and thus, their thermal behavior indicates a lower stability of these complexes in the mutant than in the WT. This was further confirmed by green gel electrophoresis, which clearly demonstrates that the thermal degradation of LHCII follows the same pattern in WT and $d g d l$, but PSI degrades faster in $d g d l$ than in WT (Fig. 2). This fact strongly suggests that the lower thermal stability of Chl $a$ excitonic CD bands (see above) is at least partially due to the faster degradation/disassembly of PSI in $d g d l$ than in WT. Faster degradation of the photosynthetic complexes in $d g d l$ is also confirmed by the temperature dependence of the $\mathrm{Chl}$ $a$ average fluorescence lifetime above $45^{\circ} \mathrm{C}$ (Fig. 4). This dependence is rather similar to the one observed for the $\mathrm{CD}$ bands at around $450 \mathrm{~nm}$ (Fig. 1b; Table 1) and, hence, it can be suggested that PSI degradation significantly contributes to it. These data are complementary to the observation of Guo et al. (2005) who revealed that PSI in $d g d l$ thylakoids is more susceptible to chaotropic agents and demonstrated the presence of PSI lacking LHCI and subunit PsaD, which could be detached from the core complex with mild detergents.

Changes in the thermal stability of $d g d l$ thylakoid membranes are also observed in the lipid matrix - the average fluorescence lifetime of MC540 in $d g d 1$ thylakoids remains shorter at all temperatures between 7 and $35^{\circ} \mathrm{C}$ (Fig. 5d). The shortening of the fluorescence lifetime of MC540 is due to its location in a more hydrophilic environment and indicates that the phase properties of the bulk lipids in the mutant membranes are changed in a way that hinders the incorporation of MC540. These data and the observed decreased thermal stabilities of the macrodomains and PSI are fully consistent with the results of Chen et al. (2006), demonstrating the role of galactolipids in thermotolerance of plants. These authors have shown a close correlation between the ability of plants to acquire thermal tolerance and the increase in the DGDG level and in the DGDG:MGDG ratio, while no correlation was found with the accumulation of heat-shock proteins.

The differences in the temperature dependencies of the lipid packing in WT and $d g d l$ might (at least in part) be due to the increased non-bilayer propensity of the bulk lipids in comparison to the WT. Previously, it has been shown, by means of ${ }^{31} \mathrm{P}-\mathrm{NMR}$, that non-bilayer lipid structures are present in spinach thylakoid membranes (Krumova et al. 2008b). Analogous ${ }^{31}$ P-NMR studies would provide valuable information for the phase properties of WT and mutant thylakoid membranes. However, given the fact that ${ }^{31} \mathrm{P}-\mathrm{NMR}$ measurements require isolated thylakoid membranes of 50-100 $\mathrm{mg}$ Chl content, it is not feasible with Arabidopsis.

While at $25^{\circ} \mathrm{C}$, the kinetic patterns of the electrochromic absorbance transients in $d g d l$ and WT leaves do not differ from each other, in the mutant, the membranes become permeable to ions even at $35^{\circ} \mathrm{C}$ (Fig. 6b), in contrast to WT, which becomes leaky only above $40^{\circ} \mathrm{C}$. Dependence of the membrane permeability on the lipid content of thylakoids was also demonstrated for a mutant of Arabidopsis ( $m g d 1-1$, Jarvis et al. 2000) with decreased amount of MGDG - the thylakoid membranes of $m g d 1-1$ were shown to exhibit increased conductivity at high light intensities, which resulted in inefficient operation of the xanthophyll cycle (Aronsson et al. 2008) and which further demonstrates the importance of the lipid phase behavior for the electric properties of the membrane.

\section{Conclusion}

It has become clear in this study that the DGDG deficiency substantially influences both the overall organization and functioning of the thylakoid membrane and its thermal stability. At room temperature $\left(25^{\circ} \mathrm{C}\right)$ the arrangement of the pigment-protein complexes in $d g d l$ differs from that in WT: the $\Psi$-type CD bands, originating from large macrodomains of pigment-protein complexes, including the LHCII, exhibit significantly lower amplitudes for $d g d l$. Experiments using the fluorescent lipid probe MC540 reveal differences in the packing of the lipid molecules, indicating a tighter packing or a modified surface charge density in the mutant thylakoid membranes. These alterations, together with a reduced PSI content of the membranes, albeit that they exert some well discernible effects on the energy migration and trapping properties as well as on the energization of the membranes, do not disturb dramatically the functions, at least as long as the plants are kept in a temperature range not higher than $25^{\circ} \mathrm{C}$. At moderately elevated temperatures, however, 
dramatic differences emerge, which are manifested in increased thermal susceptibilities in $d g d l$ compared to WT: the LHCII-PSII containing macrodomains disassemble, PSI complexes degrade, the excitation energy is quenched, large amounts of lipids are protruded from the membranes, and the thylakoids become leaky for ions - in all these cases, the changes occur $5-7^{\circ} \mathrm{C}$ lower in $d g d 1$ than in WT. Hence, these data strongly suggest that the lipid matrix of $d g d l$ is not able to maintain the functional state of the protein molecules at moderately elevated temperatures.

Acknowledgments The authors wish to thank Dr. Eva Selstam for providing the $d g d l$ seeds and for fruitful discussions and Mr. Milán Szabó for help with the electrochromic absorbance change measurements. This study was supported by grants from the Hungarian Fund for Basic Research (OTKA K 63252) to G.G., the Sandwich-Programme of Wageningen University, The Netherlands to S.B.K., the EU 6th Framework Programme Grant MRTN-CT-2005-019481 to H.v.A. and S.B.K. and the 7th Framework Programme Grant MC ITN 238017 "HARVEST" to H.v.A. and G.G.

Open Access This article is distributed under the terms of the Creative Commons Attribution Noncommercial License which permits any noncommercial use, distribution, and reproduction in any medium, provided the original author(s) and source are credited.

\section{References}

Aronsson H, Schottler MA, Kelly AA, Sundqvist C, Dörmann P, Karim S, Jarvis P (2008) Monogalactosyldiacylglycerol deficiency in Arabidopsis affects pigment composition in the prolamellar body and impairs thylakoid membrane energization and photoprotection in leaves. Plant Physiol 148:580-592. doi: 10.1104/pp.108.123372

Barzda V, Mustárdy LA, Garab G (1994) Size dependency of circular dichroism in macroaggregates of photosynthetic pigment-protein complexes. Biochemistry 33:10837-10841. doi:10.1021/ bi00201a034

Ben-Shem A, Frolow F, Nelson N (2003) Crystal structure of plant photosystem I. Nature 426:630-635. doi:10.1038/nature02200

Borst JW, Hink MA, van Hoek A, Visser AJWG (2005) Effects of refractive index and viscosity on fluorescence and anisotropy decays of enhanced cyan and yellow fluorescent proteins. J Fluoresc 15:153-160. doi:10.1007/s10895-005-2523-5

Broess K, Trinkunas G, van der Weij-de Wit CD, Dekker JP, van Hoek A, van Amerongen H (2006) Excitation energy transfer and charge separation in photosystem II membranes revisited. Biophys J 91:3776-3786. doi:10.1529/biophysj.106.085068

Broess K, Trinkunas G, van Hoek A, Croce R, van Amerongen H (2008) Determination of the excitation migration time in photosystem II. Consequences for the membrane organization and charge separation parameters. Biochim Biophys Acta 1777:404-409. doi:10.1016/j.bbabio.2008.02.003

Broess K, Borst JW, van Amerongen H (2009) Applying two-photon excitation fluorescence lifetime imaging microscopy to study photosynthesis in plant leaves. Photosynth Res 100:89-96. doi: 10.1007/s11120-009-9431-5

Büchel C, Garab G (1995) Electrochromic absorbance changes in the chlorophyll-c-containing alga Pleurochloris meiringensis (Xanthophyceae). Photosynth Res 43:49-56. doi:10.1007/ BF00029462
Chen J, Burke J, Xin Z, Xu C, Velten J (2006) Characterization of the Arabidopsis thermosensitive mutant atts 02 reveals an important role for galactolipids in thermotolerance. Plant Cell Environ 29:1437-1448. doi:10.1111/j.1365-3040.2006.01527.x

Chitnis PR (2001) Photosystem I: function and physiology. Annu Rev Plant Physiol Plant Mol Biol 52:593-626. doi:10.1146/annurev. arplant.52.1.593

Croce R, Dorra D, Holzwarth AR, Jennings RC (2000) Fluorescence decay and spectral evolution in intact photosystem I of higher plants. Biochemistry 39:6341-6348. doi:10.1021/bi992659r

Cseh Z, Rajagopal S, Tsonev T, Busheva M, Papp E, Garab G (2000) Thermooptic effect in chloroplast thylakoid membranes. Thermal and light stability of pigment arrays with different levels of structural complexity. Biochemistry 39:15250-15257. doi: 10.1021/bi001600d

De Bianchi S, Dall'Osto L, Tognon G, Morosinotto T, Bassi R (2008) Minor antenna proteins CP24 and CP26 affect the interactions between photosystem II subunits and the electron transport rate in grana membranes of Arabidopsis. Plant Cell 20:1012-1028. doi: $10.1105 /$ tpc. 107.055749

De Voe H (1965) Optical properties of molecular aggregates. II. Classical theory of the refraction, absorption, and optical activity of solutions and crystals. J Chem Phys 43:3199-3208. doi: 10.1063/1.1697294

Dekker JP, Boekema EJ (2005) Supramolecular organization of thylakoid membrane proteins in green plants. Biochim Biophys Acta 1706:12-39. doi:10.1016/j.bbabio.2004.09.009

Digris AV, Skakun VV, Novikov EG, van Hoek A, Claiborne A, Visser AJWG (1999) Thermal stability of a flavoprotein assessed from associative analysis of polarized time-resolved fluorescence spectroscopy. Eur Biophys J 28:526-531. doi:10.1007/s0024900 50235

Dobrikova AG, Várkonyi Z, Krumova SB, Kovács L, Kostov GK, Todinova SJ, Busheva MC, Taneva SG, Garab G (2003) Structural rearrangements in chloroplast thylakoid membranes revealed by differential scanning calorimetry and circular dichroism spectroscopy. Thermo-optic effect. Biochemistry 42:11272-11280. doi:10.1021/bi034899j

Dörmann P, Hoffmann-Benning S, Balbo I, Benning C (1995) Isolation and characterization of an Arabidopsis mutant deficient in the thylakoid lipid digalactosyl diacylglycerol. Plant Cell 7:1801-1810

Douce R, Joyard J (1996) Biosynthesis of thylakoid membrane lipids. In: Ort DR, Yacum CF (eds) Advances in photosynthesis/ oxygenic photosynthesis: the light reactions. Kluwer, Dordrecht, pp 69-101. doi:10.1007/0-306-48127-8

Gabashvili IS, Menikh A, Segui J, Fragata M (1998) Protein structure of photosystem II studied by FT-IR spectroscopy. Effect of digalactosyldiacylglycerol on the tyrosine side chain residues. J Mol Struct 444:123-133. doi:10.1016/S0022-2860(97)00367-0

Garab G (1996) Linear and circular dichroism. In: Amesz J, Hoff AJ (eds) Biophysical techniques in photosynthesis. Kluwer, Dordrecht, pp $11-40$

Garab G, Mustárdy L (1999) Role of LHCII-containing macrodomains in the structure, function and dynamics of grana. Aust $\mathbf{J}$ Plant Physiol 26:649-658

Garab G, van Amerongen H (2009) Linear dichroism and circular dichroism in photosynthesis research. Photosynth Res 101:135-146. doi:10.1007/s11120-009-9424-4

Garab G, Sanchez Bargos AA, Zimányi L, Faludi-Dániel A (1983) Effect of $\mathrm{CO}_{2}$ on the organization of thylakoids in leaves of higher plants. FEBS Lett 154:323-327. doi:10.1016/00145793(83)80175-6

Garab G, Kieleczawa J, Sutherland JC, Bustamante C, Hind G (1991) Organization of pigment-protein complexes into macrodomains in the thylakoid membranes of wild type and chlorophyll $b$-less mutant of barley as revealed by circular dichroism. 
Photochem Photobiol 54:273-281. doi:10.1111/j.1751-1097. 1991.tb02016.x

Garab G, Lohner K, Laggner P, Farkas T (2000) Self-regulation of the lipid content of membranes by non-bilayer lipids: a hypothesis. Trends Plant Sci 5:489-494. doi:10.1016/S1360-1385(00) 01767-2

Georgakopoulou S, van der Zwan G, Bassi R, van Grondelle R, van Amerongen $\mathrm{H}$, Croce $\mathrm{R}$ (2007) Understanding the changes in the circular dichroism of light harvesting complex II upon varying its pigment composition and organization. Biochemistry 46:4745-4754. doi:10.1021/bi062031y

Gilmore AM, Hazlett TL, Debrunner PG, Govindjee (1996) Photosystem II chlorophyll $a$ fluorescence lifetimes and intensity are independent of the antenna size differences between barley wildtype and chlorina mutants: photochemical quenching and xanthophyll cycle dependent non-photochemical quenching of fluorescence. Photosynth Res 48:171-187. doi:10.1007/BF00041007

Gounaris K, Brain ARR, Quinn PJ, Williams WP (1984) Structural reorganization of chloroplast thylakoid membranes in response to heat-stress. Biochim Biophys Acta 766:198-208. doi: 10.1016/0005-2728(84)90232-9

Guo J, Zh Zhang, Bi Y, Yang W, Xu Y, Zhang L (2005) Decreased stability of photosystem I in $d g d 1$ mutant of Arabidopsis thaliana. FEBS Lett 579:3619-3624. doi:10.1016/j.febslet.2005. 05.049

Härtel H, Lokstein H, Dörmann P, Grimm B, Benning C (1997) Changes in the composition of the photosynthetic apparatus in the galactolipid-deficient $d g d 1$ mutant of Arabidopsis thaliana. Plant Physiol 115:1175-1184

Härtel H, Lokstein H, Dörmann P, Trethewey RN, Benning C (1998) Photosynthetic light utilization and xanthophyll cycle activity in the galactolipid deficient $d g d 1$ mutant of Arabidopsis thaliana. Plant Physiol Biochem 36:407-417. doi:10.1016/S09819428(98)80204-0

Harwood JL (1998) Involvement of chloroplast lipids in the reaction of plants submitted to stress. In: Siegenthaler PA, Murata N (eds) Advances in photosynthesis. Lipids in photosynthesis. Kluwer, Dordrecht, pp 287-302

Hendrickson L, Vlčkova A, Selstam E, Huner N, Öquist G, Hurry V (2006) Cold acclimation of the Arabidopsis $d g d 1$ mutant results in recovery from photosystem I-limited photosynthesis. FEBS Lett 580:4959-4968. doi:10.1016/j.febslet.2006.07.081

Ihalainen JA, Jensen PE, Haldrup A, van Stokkum IHM, van Grondelle R, Scheller HV, Dekker JP (2002) Pigment organization and energy transfer dynamics in isolated photosystem I (PSI) complexes from Arabidopsis thaliana depleted of the PSIG, PSI-K, PSI-L, or PSI-N subunit. Biophys J 83:2190-2201. doi:10.1016/S0006-3495(02)73979-9

Ihalainen JA, van Stokkum IHM, Gibasiewicz K, Germano M, van Grondelle R, Dekker JP (2005) Kinetics of excitation trapping in intact photosystem I of Chlamydomonas reinhardtii and Arabidopsis thaliana. Biochim Biophys Acta 1706:267-275. doi: 10.1016/j.bbabio.2004.11.007

Ivanov AG, Hendrickson L, Krol M, Selstam E, Öquist G, Hurry V, Huner NPA (2006) Digalactosyl-diacylglycerol deficiency impairs the capacity for photosynthetic intersystem electron transport and state transitions in Arabidopsis thaliana due to photosystem I acceptor-side limitations. Plant Cell Physiol 47:1146-1157. doi:10.1093/pcp/pcj089

Jarvis P, Dörmann P, Peto CA, Lutes J, Benning C, Chory J (2000) Galactolipid deficiency and abnormal chloroplast development in the Arabidopsis MGD synthase 1 mutant. Proc Natl Acad Sci USA 97:8175-8179. doi:10.1073/pnas.100132197

Junge W (1977) Membrane potentials in photosynthesis. Annu Rev Plant Physiol 128:503-536. doi:10.1146/annurev.pp.28.060177. 002443
Kovács L, Damkjar J, Kereiche S, Ilioaia C, Ruban AV, Boekema EJ, Jansson S, Horton P (2006) Lack of the light-harvesting complex CP24 affects the structure and function of the grana membranes of higher plant chloroplasts. Plant Cell 18:3106-3120. doi: 10.1105/tpc.106.045641

Krumova SB, Koehorst RBM, Bóta A, Páli T, van Hoek A, Garab G, van Amerongen $\mathrm{H}$ (2008a) Temperature dependence of the lipid packing in thylakoid membranes studied by time- and spectrally resolved fluorescence of Merocyanine 540. Biochim Biophys Acta 1778:2823-2833. doi:\#10.1016/j.bbamem.2008.09.007

Krumova SB, Dijkema C, de Waard P, Van As H, Garab G, van Amerongen H (2008b) Phase behavior of phosphatidylglycerol in spinach thylakoid membranes as revealed by ${ }^{31} \mathrm{P}-\mathrm{NMR}$. Biochim Biophys Acta 1778:997-1003. doi:10.1016/j.bbamem. 2008.01.004

Lelkes PI, Miller IR (1980) Perturbations of membrane structure by optical probes: I. Location and structural sensitivity of Merocyanine 540 bound to phospholipid membranes. J Membr Biol 52:1-15. doi:10.1007/BF01869001

Liu ZF, Yan HC, Wang KB, Kuang TY, Zhang JP, Gui LL, An XM, Chang WR (2004) Crystal structure of spinach major lightharvesting complex at 2.72 angstrom resolution. Nature 428:287-292. doi:10.1038/nature02373

Loll B, Kern J, Sänger W, Zouni A, Biesiadka J (2005) Towards complete cofactor arrangement in the $3.0 \AA$ resolution structure of photosystem II. Nature 438:1040-1044. doi:10.1038/ nature 04224

Loll B, Kern J, Sänger W, Zouni A, Biesiadka J (2007) Lipids in photosystem II: interactions with protein and cofactors. Biochim Biophys Acta 1767:509-519. doi:10.1016/j.bbabio.2006.12.009

Mitchell P (1966) Chemiosmotic coupling in oxidative and photosynthetic phosphorylation. Biol Rev Camb Philos Soc 41:445-502

Mustárdy L, Garab G (2003) Granum revisited. A three-dimensional model-where things fall into place. Trends Plant Sci 8:117-122. doi:10.1016/S1360-1385(03)00015-3

Novikov EG, van Hoek A, Visser AJWG, Hofstraat JW (1999) Linear algorithms for stretched exponential decay analysis. Opt Commun 166:189-198. doi:10.1016/S0030-4018(99)00262-X

$\mathrm{Nu} \beta$ berger S, Dörr K, Wang DN, Kühlbrandt W (1993) Lipid-protein interactions in crystals of plant light-harvesting complex. J Mol Biol 234:347-356. doi:10.1006/jmbi.1993.1591

Roelofs TA, Lee CI, Holzwarth AR (1992) Global target analysis of picosecond chlorophyll fluorescence kinetics from pea chloroplasts: a new approach to the characterization of the primary processes in photosystem II $\alpha$-units and $\beta$-units. Biophys $\mathbf{J}$ 61:1147-1163. doi:10.1016/S0006-3495(92)81924-0

Sakurai I, Mizusawa N, Wada H, Sato N (2007) Digalactosyldiacylglycerol is required for stabilization of the oxygen-evolving complex in photosystem II. Plant Physiol 145:1361-1370. doi: 10.1104/pp.107.106781

Slavov C, Ballottari M, Morosinotto T, Bassi R, Holzwarth AR (2008) Trap-limited charge separation kinetics of photosystem I complexes from higher plant. Biophys J 94:3601-3612. doi: 10.1529/biophysj.107.117101

Smith PJ, Peterson S, Masters VM, Wydrzynski T, Styring S, Krausz E, Pace RJ (2002) Magneto-optical measurements of the pigments in fully active photosystem II core complexes from plants. Biochemistry 41:1981-1989. doi:10.1021/bi0111202

Somsen OJG, van Grondelle R, van Amerongen H (1996) Spectral broadening of interacting pigments: polarized absorption by photosynthetic proteins. Biophys J 71:1934-1951. doi:10.1016/ S0006-3495(96)79392-X

Tinoco I (1962) Theoretical aspects of optical activity. 2. Polymers. Adv Chem Phys 4:113-160. doi:10.1002/9780470143506.ch3

Turconi S, Weber N, Schweitzer G, Strotmann H, Holzwarth AR (1994) Energy transfer and charge separation kinetics in 
photosystem I. 2. Picosecond fluorescence study of various PS I particles and light-harvesting complex isolated from higher plants. Biochim Biophys Acta 1187:324-334. doi:10.1016/ S0006-3495(93)81552-2

van Metter RL (1977) Excitation energy transfer in the lightharvesting chlorophyll $a / b$ protein. Biochim Biophys Acta 462:642-658. doi:10.1016/0005-2728(77)90107-4

van Oort B, Amunts A, Borst JW, van Hoek A, Nelson N, van Amerongen H, Croce R (2008) Picosecond fluorescence of intact and dissolved PSI-LHCI crystals. Biophys J 95:5851-5861. doi: 10.1529/biophysj.108.140467

van Oort B, Alberts M, de Bianchi S, Dall'Osto L, Bassi R, Trinkunas G, Croce R, van Amerongen H (2010) Effect of antennadepletion in photosystem II on excitation energy transfer in Arabidopsis thaliana. Biophys J 98:922-931. doi:10.1016/ j.bpj.2009.11.012

Vasile'v S, Wiebe S, Bruce D (1998) Non-photochemical quenching of chlorophyll fluorescence in photosynthesis. 5-Hydroxy-1,4naphthoquinone in spinach thylakoids as a model for antenna based quenching mechanisms. Biochim Biophys Acta 1363:147-156. doi:10.1016/S0005-2728(97)00096-0

Visser NV, Westphal AH, van Hoek A, van Mierlo CPM, Visser AJWG, van Amerongen H (2008) Tryptophan-tryptophan energy migration as a tool to follow apoflavodoxin folding. Biophys $\mathbf{J}$ 95:2462-2469. doi:10.1529/biophysj.108.132001

Williams WP (1998) The physical properties of thylakoid membrane lipids and their relation to photosynthesis. In: Siegenthaler PA, Murata N (eds) Advances in photosynthesis. Lipids in photosynthesis. Kluwer, Dordrecht, pp 103-118

Witt HT (1979) Energy conversion in the functional membrane of photosynthesis. Analysis by light pulse and electric pulse methods. The central role of the electric field. Biochim Biophys Acta 505:355-427. doi:10.1016/0304-4173(79)90008-9

Yan H, Zhang P, Wang C, Liu ZH, Chang W (2007) Two lutein molecules in LHCII have different conformations and functions: insights into the molecular mechanism of thermal dissipation in plants. Biochem Biophys Res Commun 355:457-463. doi: 10.1016/j.bbrc.2007.01.172 